\title{
NOUVELLE
}

\section{Fluctuations aléatoires dans le métabolisme et la croissance cellulaires}

Philippe Nghe ${ }^{1,2}$, Sarah Boulineau ${ }^{1}$, Sander J. Tans ${ }^{1}$

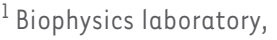 \\ AMOLF (the institute for atomic and molecular physics), \\ Science Park, 1098 XG Amsterdam, Pays-Bas ; \\ ${ }^{2}$ Laboratoire de biochimie, \\ ESPCI (École supérieure de physique chimie industrielle), \\ 10, rue Vauquelin, 75005 Paris, France. \\ philippe.nghe@espci.fr
}

\section{Fluctuations d'origine thermique}

\section{à l'échelle cellulaire}

Entre l'observation de Brown en 1827 et la démonstration d'Einstein en 1905, il fallut presqu'un siècle pour que les mouvements incessants des grains de pollens en solution soient interprétés comme une conséquence de l'agitation thermique aux petites échelles plutôt que comme un caractère vivant. En 1944, le physicien Erwin Schrödinger remet la question au goût du jour [1], en constatant que de telles fluctuations pourraient altérer les chromosomes, récemment identifiés comme étant la source de l'organisation cellulaire. Étant donné le petit nombre de ces derniers, il se demande à quel point ceci pourrait avoir des conséquences physiologiques.

C'est seulement durant la dernière décennie que cette idée fut vérifiée, grâce à une quantification suffisamment précise et systématique de la production de protéines et d'ARN par microscopie de fluorescence sur des cellules uniques [2, 3]. Les fluctuations d'origine thermique affectant non seulement les trajectoires des molécules, mais aussi les interactions entre les complexes moléculaires de la transcription et de la traduction, elles sont à l'origine de variations significatives du niveau d'expression des gènes. Ces variations, qui représentent typiquement $10 \%$ à $30 \%$ du niveau moyen, peuvent affecter d'autres gènes régulés en aval, et s'additionner à travers les cascades de signalisation [4]. Cependant, il n'est pas a priori évident qu'elles puissent déterminer l'état des cellules à un niveau véritablement physiologique, tel que le métabolisme ou la croissance.

\section{Quantifier les fluctuations à l'échelle} de la cellule unique

Nous avons combiné microscopie en time-lapse, analyse d'image de cellules uniques, analyse statistique et modélisation mathématique pour montrer comment et à quel point les fluctuations d'expression des gènes peuvent affecter la croissance de bactéries Escherichia coli [5].

La microscopie time-lapse consiste simplement à capturer des images de colonies de bactéries à des intervalles de temps réguliers. Les bactéries, inoculées à faible densité, croissent en monocouches sur une surface de polyacrylamide imbibée d'un milieu de culture [6]. Nous avons enregistré, à raison d'environ une image par minute et à grossissement 100X, les divisions cellulaires durant 9 générations à partir d'une cellule unique, soit de l'ordre de 500 bactéries après plusieurs heures. Un logiciel maison nous a permis de déterminer automatiquement le contour des cellules individuelles à partir d'images de contraste de phase (Figure 1A), puis de mesurer avec précision la longueur de chaque cellule (Figure 1B). Le taux de croissance instantané est ensuite calculé par la dérivée du logarithme de la longueur (Figure 1C). L'acquisition d'images de fluorescence permet de mesurer simultanément l'expression de protéines fluorescentes associées à des gènes d'intérêt (Figure ID), tels que ceux codant pour des enzymes métaboliques.

Nous obtenons alors, pour chaque lignée, l'expression de gènes d'intérêt et le taux de croissance cellulaire, avec plusieurs mesures par cycle cellulaire. Pour interpréter ces données, nous avons utilisé les corrélations calculées avec différents décalages temporels entre les signaux. En effet, une corrélation usuelle entre signaux simultanés ne permet pas d'identifier la causalité entre deux signaux $X$ et $Y$, sachant que $X$ peut causer $Y$, ou l'inverse, ou bien la corrélation peut provenir d'un phénomène tiers $Z$, ou encore d'un mélange entre ces différentes possibilités. La prise en compte de délais temporels entre les signaux résout en grande partie ce problème, car elle permet de discriminer de nombreux scénarios de causalité parmi un ensemble de modèles envisagés [7].

Considérons le taux de croissance $\mu$ des cellules (Figure IC) et la concentration $\varepsilon$ de la $\beta$-galactosidase (Figure ID), enzyme catabolique nécessaire à la croissance sur un milieu ayant pour seule source de carbone le lactose. Pour un décalage temporel donné $\Delta t$, on calcule le coefficient de corrélation $\mathrm{R}(\Delta \mathrm{t})$ entre les signaux $\mu(t)$ et $\varepsilon(t+\Delta t)$ (Figure $1 \varepsilon$ ). On obtient ainsi une fonction $\mathrm{R}(\Delta \mathrm{t})$ dont l'asymétrie informe sur l'existence de délais entre les signaux (Figure IF), ces délais étant ici typiquement d'un cycle cellulaire, soit environ 1 heure. 


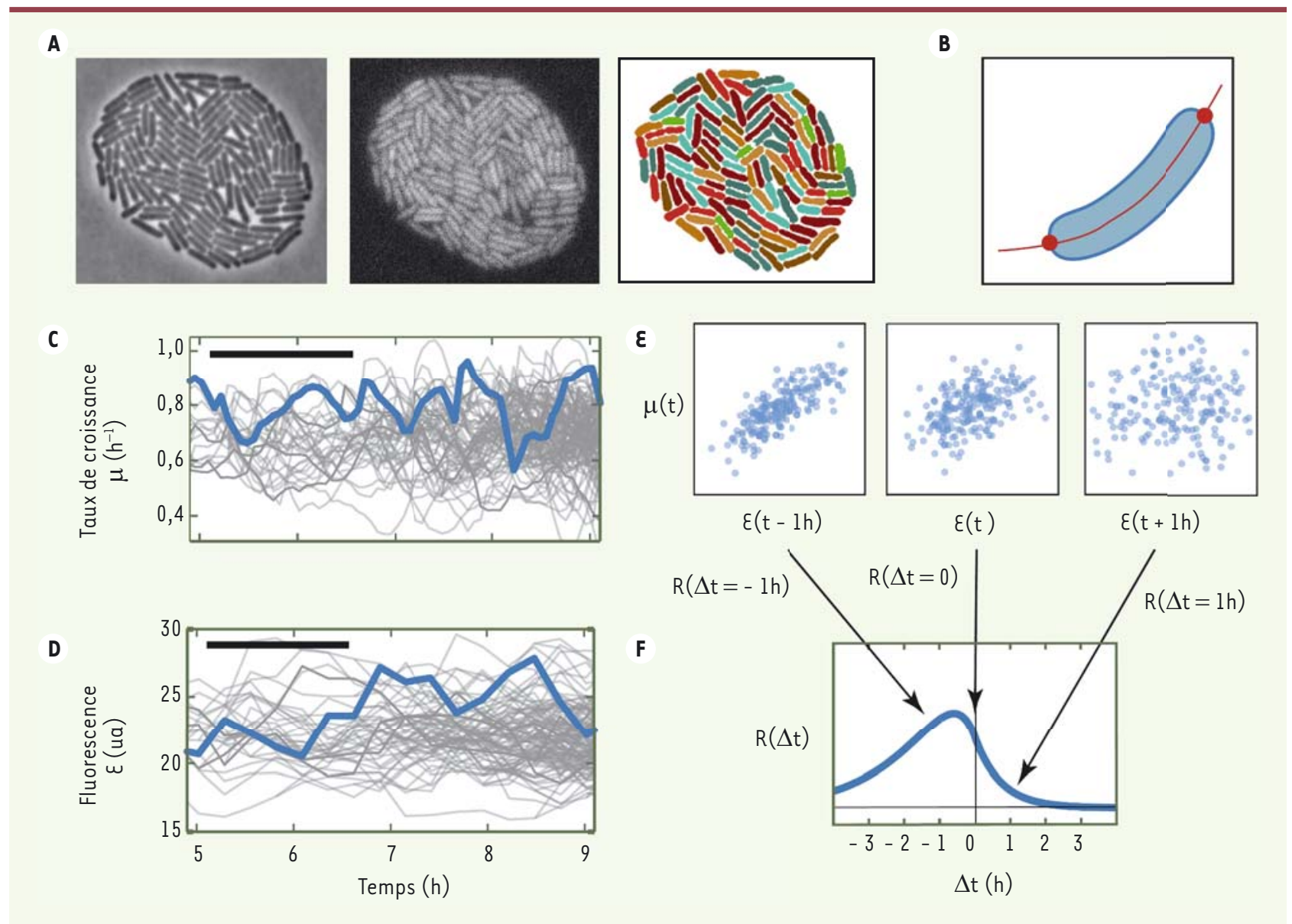

Figure 1. Analyse des fluctuations de croissance et d'expression enzymatique. A. Des images de colonies bactériennes sont acquises à intervalles régulier, en contraste de phase (gauche), en fluorescence (milieu), les bactéries individuelles étant ensuite détectées automatiquement (droite) d'après l'image de contraste de phase. B. Pour chaque bactérie détectée, on mesure sa longueur. C. Cette longueur est utilisée pour calculer le taux de croissance de chaque lignée. $D$. La fluorescence est également extraite par cellule individuelle puis reconstituée par lignée. $\varepsilon$. Pour chaque décalage temporel $\Delta$ t entre les signaux représentés en $C$ et $D$, on extrait le nuage de points $\mu(t)$ en fonction de $\varepsilon(t+\Delta t)$, obtenu pour toutes les valeurs de $\mathrm{t}$ à $\Delta \mathrm{t}$ fixé, et on calcule le coefficient de corrélation correspondant $\mathrm{R}(\Delta \mathrm{t})$. $F$. On représente finalement la fonction $\mathrm{R}(\Delta \mathrm{t})$, qui, dans cet exemple, a des valeurs plus élevées aux décalages temporels négatifs entre $\mu$ et $\varepsilon$, indiquant que les fluctuations de concentration de l'enzyme sont typiquement en retard sur celles de la croissance.

Modes de propagation et fluctuations de croissance

On a mesuré que lorsque la concentration de l'enzyme catabolique est maintenue à un niveau limitant pour le métabolisme, les fluctuations du taux de croissance tendent à apparaître plus tard que celles du taux de production de l'enzyme. À l'opposé, lorsqu'une concentration saturante de l'enzyme est maintenue, elle tend à fluctuer en retard comparé aux fluctuations du taux de croissance (Figure IF). L'hypothèse explicative la plus élémentaire est qu'il existe différents modes de transmission des fluctuations dans les cellules, modes qui dominent plus ou moins les corrélations en fonction de la concentration moyenne de l'enzyme: (1) les fluctuations de croissance causent en toute situation des fluctuations de concentrations de l'enzyme, (2) si l'enzyme est limitante, ses fluctuations causent des fluctuations de croissance.

Nous avons effectué une comparaison quantitative des corrélations croisées temporelles et d'un modèle mathématique utilisant les relations connues entre croissance et expression enzymatique. Nous avons utilisé une approche phé- noménologique, dans laquelle les différentes variables réagissent de manière linéaire aux variations des autres. Un tel modèle, malgré la simplification extrême qu'il représente par rapport aux processus cellulaires, explique avec une grande fidélité les corrélations mesurées en utilisant très peu de paramètres. Le résultat essentiel de cette analyse est que les fluctuations se décomposent en modes élémentaires de transmission, qui se superposent les uns aux autres dans des proportions variables selon le degré de limitation de l'enzyme, le gène considéré et le milieu nutritif. 
A

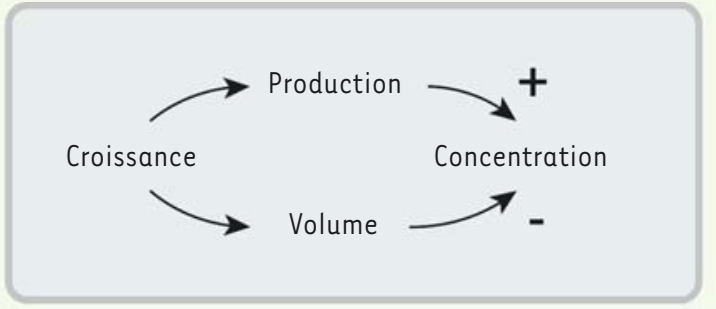

B

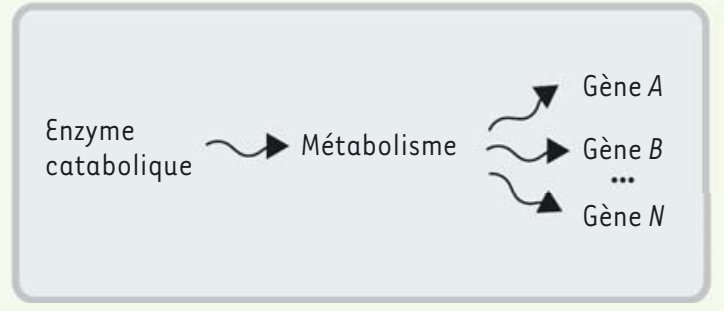

C

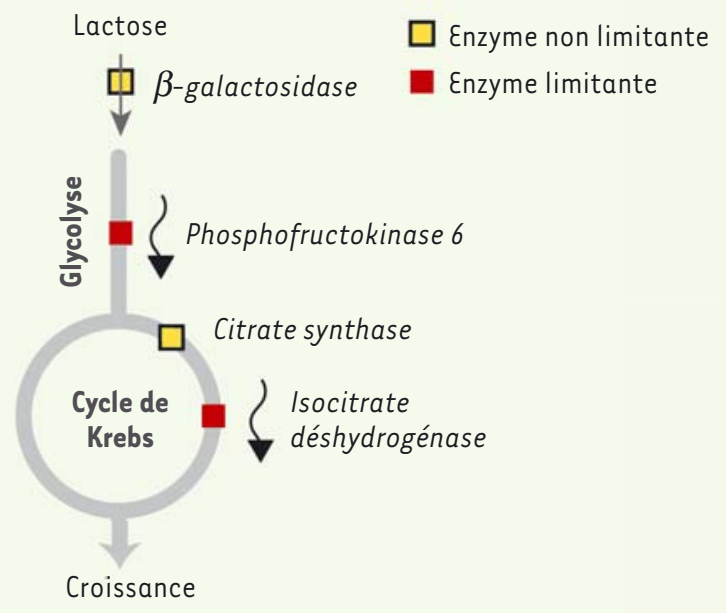

Figure 2. Mécanismes de transmission des fluctuations dans la cellule. A. Une augmentation temporaire de croissance cause simultanément une augmentation de la production de protéines et du volume, ce dernier contribuant à équilibrer l'excès de production par dilution. $B$. Les enzymes cataboliques limitant le flux métabolique peuvent engendrer des fluctuations corrélées dans tous les gènes, et ce indépendamment de tout lien de régulation. C. Durant la croissance sur le lactose, certaines enzymes telles que la phosphofructokinase ou l'isocitrate deshydrogénase sont limitantes pour le métabolisme. Leurs fluctuations se transmettent alors à la croissance cellulaire. À l'opposé, les fluctuations d'autres enzymes appartenant aux mêmes voies métaboliques, mais non limitantes, telles que la $\beta$-galactosidase ou la citrate synthase, n'ont pas d'impact mesurable sur la croissance.

Outre des confirmations de la causalité par perturbations externes, nous avons testé les modes de transmissions prédits par notre modèle en effectuant plusieurs modifications génétiques. Nous avons ainsi pu vérifier qu'une augmentation temporaire de croissance cause à la fois une augmentation de production des protéines et une augmentation de volume, qui peuvent se compenser pour maintenir la concentration protéique constante (Figure 2A). Par ailleurs, puisque les fluctuations d'une enzyme se propagent à la croissance, qui elle-même perturbe l'expression d'autres gènes, les fluctuations d'un seul gène métabolique causent des fluctuations d'expression d'autres gènes sans lien de régulation, par la seule modulation de l'activité métabolique (Figure 2B). Enfin, nous avons vérifié sur des gènes de la glycolyse et du cycle de Krebs, que les fluctuations de leur expression engendrent des fluctuations de croissance si et seulement s'ils sont limitants, et ce indépendamment du fait que les enzymes correspondantes soient consécutives dans la chaîne métabolique (Figure 2C).

\section{Conclusion}

Nous pouvons finalement répondre à I'interrogation de Schrödinger: l'agitation thermique à l'échelle des molécules biologiques a des conséquences observables à un niveau physiologique, en particulier sur la croissance cellulaire. Ainsi, la cellule traverse en permanence une diversité d'états métaboliques, diversité qui prend une importance particulière lors d'événements de différenciation [8], notamment en réponse à des stress [9] ou des changements environnementaux [10]. Paradoxalement, la sensibilité du vivant aux fluctuations d'origine physique semble être une contrepartie, inévitable à petite échelle, au haut degré d'organisation et d'interdépendance dans la cellule. $\diamond$

Random fluctuations, metabolism and growth at the single-cell level

\section{LIENS D'INTÉRÊT}

Les auteurs déclarent n'avoir aucun lien d'intérêt concernant les données publiées dans cet article.

\section{RÉFÉRENCES}

1. Schrödinger $\varepsilon$. What is life? With mind and matter and autobiographical sketches. New York: Cambridge University Press, 1992.

2. Elowitz MB, Levine AJ, Siggia ED, Swain PS. Stochastic gene expression in a single cell. Science 2002 ; 297 : 1183-6.

3. Cai L, Friedman N, Xie XS. Stochastic protein expression in individual cells at the single molecule level. Nature $2006 ; 440$ : 358-62.

4. Pedraza JM, van Oudenaarden A. Noise propagation in gene networks. Science 2005 ; 307 : 1965-9.

5. Kiviet DJ, Nghe P, Walker N, et al. Stochasticity of metabolism and growth at the single-cell level. Nature 2014 ; 514 : 376-9.

6. Nghe P, Boulineau S, Gude S, et al. Microfabricated polyacrylamide devices for the controlled culture of growing cells and developing organisms. PloS One 2013 ; 8 : e75537.

7. Dunlop MJ, Cox RS, Levine JH, et al. Regulatory activity revealed by dynamic correlations in gene expression noise. Nat Genet 2008 ; 40 : 1493-8.

8. Chang $\mathrm{HH}$, Hemberg M, Barahona $M$, et al. Transcriptome-wide noise controls lineage choice in mammalian progenitor cells. Nature 2008 ; 453 : 544-7.

9. Locke JC, Young JW, Fontes M, et al. Stochastic pulse regulation in bacterial stress response. Science 2011 ; 334 : 366-9.

10. Boulineau S, Tostevin F, Kiviet DJ, et al. Single-cell dynamics reveals sustained growth during diauxic shifts. PloS One 2013 ; 8 : e61686.

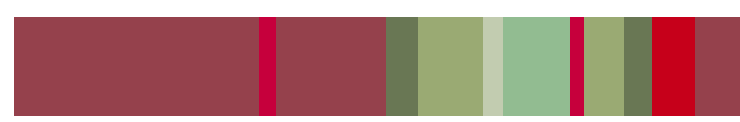

